"Neural networks application in managing the energy efficiency of industrial enterprise"

\begin{tabular}{|c|c|}
\hline AUTHORS & Svitlana Klepikova (D https://orcid.org/0000-0002-3218-943X \\
\hline ARTICLE INFO & $\begin{array}{l}\text { Svitlana Klepikova (2018). Neural networks application in managing the energy } \\
\text { efficiency of industrial enterprise. Neuro-Fuzzy Modeling Techniques in } \\
\text { Economics, } 7(1), 62-73 \text {. doi:10.21511/nfmte.7.2018.04 }\end{array}$ \\
\hline DOI & http://dx.doi.org/10.21511/nfmte.7.2018.04 \\
\hline RELEASED ON & Thursday, 18 July 2019 \\
\hline RECEIVED ON & Tuesday, 17 July 2018 \\
\hline \multirow[t]{2}{*}{ ACCEPTED ON } & Tuesday, 14 August 2018 \\
\hline & $(\mathrm{cc}) \mathrm{EY}$ \\
\hline LICENSE & $\begin{array}{l}\text { This work is licensed under a Creative Commons Attribution } 4.0 \text { International } \\
\text { License }\end{array}$ \\
\hline JOURNAL & "Neuro-Fuzzy Modeling Techniques in Economics" \\
\hline ISSN PRINT & $2306-3289$ \\
\hline ISSN ONLINE & $2415-3516$ \\
\hline PUBLISHER & LLC "Consulting Publishing Company "Business Perspectives" \\
\hline FOUNDER & $\begin{array}{l}\text { State Higher Educational Establishment "Kyiv National Economic University } \\
\text { named after Vadym Hetman" }\end{array}$ \\
\hline & $\begin{array}{l}= \pm= \\
= \pm=\end{array}$ \\
\hline NUMBER OF REFERENCES & NUMBER OF FIGURES \\
\hline 19 & 4 \\
\hline
\end{tabular}

(C) The author(s) 2023. This publication is an open access article. 


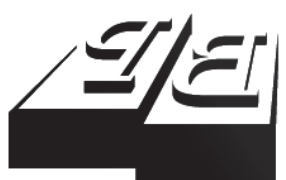

BUSINESS PERSPECTIVES

Publisher

LLC "CPC "Business Perspectives" Hryhorii Skovoroda lane, 10, Sumy, 40022, Ukraine www.businessperspectives.org

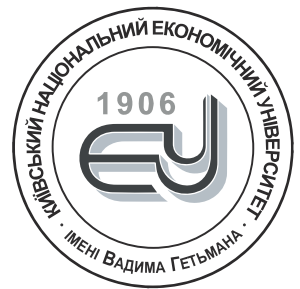

KNEU named after Vadym Hetman

Founder

State Higher Educational

Establishment "Kyiv National

Economic University named after

Vadym Hetman",

Prospect Peremogy, 54/1,

Kyiv, 03057, Ukraine

https://kneu.edu.ua/

Received on: 17th of

July, 2018

Accepted on: 14th of

August, 2018

\section{(c) Svitlana Klepikova, 2018}

Svitlana Klepikova, Senior Instructor of Department of International Business and Finance National Technical University

"Kharkiv Polytechnic Institute", Ukraine.

\section{(c) (i)}

This is an Open Access article, distributed under the terms of the Creative Commons Attribution 4.0 International license, which permits unrestricted re-use, distribution, and reproduction in any medium, provided the original work is properly cited.

\title{
NEURAL NETWORKS APPLICATION IN MANAGING THE ENERGY EFFICIENCY OF INDUSTRIAL ENTERPRISE
}

\begin{abstract}
The article is devoted to the creation of a method for using of neural networks approach in solving problems of energy efficiency management at the industrial enterprise. The method allows to obtain an approximate expected value of the energy intensity of production, depending on the values of the main factors affecting it. The multilayer perceptron was chosen as the type of neural network, synthesis of which was carried out by using the genetic algorithm.

When sampling for the synthesis of a neural network, we used the results that were obtained by means of a priori ranking, correlation and regression analysis based on the statistical data of industrial enterprises in machine-building profile.

The recommendations of the use of the method and the application of its results in the practical implementation at the industrial enterprise are given. Calculations based on the aforementioned method ensured a high precision of prediction of energy intensity values for industrial enterprises that were included in the sample during the synthesis of the neural network, and an acceptable error while testing on industrial enterprises from a test sample.
\end{abstract}

Keywords

JEL Classification energy efficiency of the industrial enterprise, energy intensity, a priori ranking, correlation and regression analysis, neural network, genetic algorithm, multilayer perceptron

\footnotetext{
C45, C61, Q40, M11
}

\section{С.В. Клепікова (Україна)}

\section{ЗАСТОСУВАННЯ НЕЙРОННИХ МЕРЕЖ В УПРАВЛІННІ ЕНЕРГОЕФЕКТИВНІСТЮ ПІДПРИЕМСТВА}

\begin{abstract}
Анотація
Стаття присвячена розробці методики використання нейронних мереж при вирішенні завдань управління енергоефективністю підприємства, що дозволяє отримати наближене очікуване значення енергоємності виробництва в залежності від значень основних факторів які впливають на неї. Типом нейронної мережі обрано багатошаровий прямоспрямований перцептрон, синтез якого здійснений із застосуванням методу генетичного алгоритму. При формуванні вибірки для синтезу нейронної мережі використані результати, отримані методом апріорного ранжирування і кореляційно-регресійного аналізу за статистичними даними промислових підприємств машинобудівного профілю.

Наведено рекомендації щодо використання методики і застосування іiі результатів при практичній імплементації на підприємстві. Проведені на ії основі розрахунки забезпечили високу точність прогнозування значень енергоємності для підприємств, які були включені до вибірки при синтезі нейронної мережі, і прийнятну похибку при перевірці за підприємствами, включеними до тестової вибірки.
\end{abstract}

\section{Ключові слова}

Класифікація JEL енергоефективність підприємства, енергоємність, апріорне ранжування, кореляційно-регресійний аналіз, нейронна мережа, генетичний алгоритм, багатошаровий перцептрон 


\title{
ПРИМЕНЕНИЕ НЕЙРОННЫХ СЕТЕЙ В УПРАВЛЕНИИ ЭНЕРГОЭФФЕКТИВНОСТЬЮ ПРЕДПРИЯТИЯ
}

\begin{abstract}
Аннотация
Статья посвящена созданию методики использования нейронных сетей при решении задач управления энергоэффективностью предприятия, позволяющей получить приближенное ожидаемое значение энергоемкости производства в зависимости от значений основных факторов, влияющих на нее. В качестве нейронной сети выбран многослойный прямонаправленный перцептрон, синтез которого осуществлен применением метода генетического алгоритма.

При определении выборки для синтеза нейронной сети использованы результаты, полученные методом априорного ранжирования и корреляционно-регрессионного анализа по статистическим данным промышленных предприятий машиностроительного профиля.

Приведены рекомендации использования методики и применения ее результатов при практической реализации на предприятии. Проведенные на ее основе расчеты обеспечили высокую точность прогнозирования значений энергоемкости для предприятий, которые были включены в выборку при синтезе нейронной сети, и приемлемую погрешность при проверке по предприятиям, включенным в тестовую выборку.
\end{abstract}

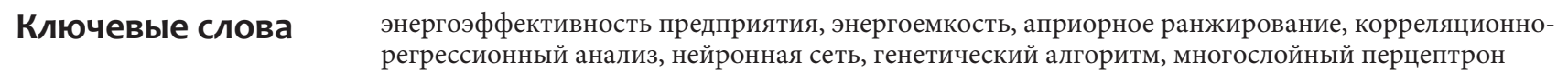

Классификация JEL C45, C61, Q40, M11

\section{ВВЕАЕНИЕ}

Развитие экономики любой страны требует рационального использования энергетических ресурсов. Для Украины, импортирующей около половины необходимых стране энергоресурсов и затрачивающей на это огромные валютные средства, повышение энергоэффективности экономики особо актуально. По оценкам экспертов, украинские предприятия затрачивают во много раз больше энергетических ресурсов на производство единицы продукции, чем их конкуренты в экономически развитых странах.

Кабинетом Министров Украины одобрена Энергетическая стратегия Украины на период до 2035 года «Безопасность, энергоэффективность, конкурентоспособность», предусматривающая достижение энергосбережения в размере 9\% по отношению к среднему энергопотреблению за период 2005-2009 гг., что сэкономит 6.5 млн тонн энергоресурсов, а общий потенциал энергосбережения в Украине к 2030 г. составит 570.3 млн тонн условного топлива [1]. Состояние и перспективы развития топливноэнергетического комплекса Украины отображены в работе [17].

В работе [2] отмечается, что наибольший удельный вес (53-58\%) в структуре потенциала энергосбережения в Украине имеет промышленность, в частности, вследствие отставания в темпах обновления оборудования, внедрения новых технологий и других факторов. При этом следует учитывать взаимосвязь повышения общегосударственной энергоэффективности конкретного предприятия с другими секторами экономики, в соответствии с «пирамидальным подходом», рекомендованным Международным энергетическим агентством (International Energy Agency, IEA, МЭА) [6]. Повышению энергоэффективности промышленного предприятия способствует научная организация управления процессом экономии энергетических ресурсов, что нашло отражение в работах $[3 ; 7 ; 13 ; 18 ; 19]$.

При решении экономических задач все в большей мере используются методы математической статистики и интеллектуальные методы управления: нечеткой логики и искусственных нейронных сетей (ИНС) [4; $8 ; 9 ; 12 ; 14 ; 16]$.

Применение вышеуказанных методов для решения задач экономики в основном относились к области финансового менеджмента: прогнозирование рынка ценных бумаг и валютных котировок, оценка финансовых, валютных и кредитных рисков, категоризация компаний и др. Для решения задач 
управления энергоэффективностью промышленного предприятия в работе [16] использовались методы математической статистики: метод априорного ранжирования и корреляционно-регрессионного анализа.

\section{1. ЦЕЛЬ ИССЛЕДОВАНИЯ}

Цель настоящей работы - разработать методику применения искусственных нейронных сетей для определения энергоемкости производства в зависимости от наиболее влияющих на нее факторов.

Для достижения поставленной цели необходимо решить следующие задачи:

- отобрать влияющие факторы, используемые в качестве входных величин нейронной сети, а также показатель энергоэффективности в качестве выходного результата;

- выбрать вид нейронной сети, ее структуру, характер активационной функции и метод обучения;

- сформировать выборку для обучения нейронной сети на основе статистических данных части предприятий (синтезированная нейронная сеть должна обеспечивать для каждого из предприятий соответствие расчетных и статистических значений);

- проверить адекватность функционирования синтезированной нейронной сети по предприятиям, не включенным в выборку, и оценить степень расхождения полученных результатов расчета со статистическими данными;

- проанализировать, в какой мере допустимо использование синтезированных ИНС;

- провести анализ и представить рекомендации, на каких этапах организации управления энергоэффективностью предприятия возможно использование ИНС;

- провести сравнительный анализ предложенного подхода на основе использования ИНС со статистическими методами, в частности априорного ранжирования и корреляционно-регрессионного анализа, для определения области их целесообразного применения.

\section{2. ИЗЛОЖЕНИЕ ОСНОВНОГО МАТЕРИАЛА}

Для повышения эффективности управления промышленным предприятием важно иметь математический аппарат, позволяющий осуществлять оценку и прогнозирование показателя энергоэффективности в зависимости от значений, влияющих на него факторов. Для этого необходимо получить математическую модель, адекватно отображающую взаимосвязи между такими факторами и показателем энергоэффективности.

Эффективный инструментарий поиска таких закономерностей предоставляет технология построения искусственной нейронной сети, которая может быть синтезирована (обучена) по статистическим данным отобранных показателей, предоставленных предприятиями.

При разработке методики синтеза нейросети используем результаты априорного ранжирования и корреляционно-регрессионного анализа десяти предприятий машиностроительного профиля, приведенные в работе [16]. Обучение проводилось с применением программы Mendel4 [11], которая реализует диплоидную версию генетического алгоритма [10], разработанную в НТУ «ХПИ» и апробированную при решении технических задач.

Для решения поставленной задачи прежде всего была создана экспертная группа из десяти специалистов энергетических цехов, подразделений технологической и экономической подготовки производства и представителей руководства предприятий. Каждому эксперту было предложено определить влияние на энергоемкость каждого из 15-ти влияющих на нее факторов $\left(X_{1}, X_{2}, \ldots . X_{15}\right)$, оценив их местом (рангом) от 1 до 15 по степени влияния в порядке убывания. С помощью коэффициента конкордации Кенделла было подтверждено определенное согласие между экспертами, а по критерию Пирсона - не случайность согласия экспертов. 
На основе присвоенных экспертами каждому из 15-ти факторов $X_{x}, x=\overline{1,15}$, оценок была построена априорная диаграмма рангов $M_{x}$ (от 1 до 15) и определены удельные веса факторов $q_{x}$ по степени их влияния на энергоемкость:

$q_{x}=\frac{2\left(15-M_{x}+1\right)}{15(15+1)}$

где $x$ - номер фактора, а сумма весов всех 15-ти факторов равна единице:

$\sum_{x=1}^{15} q_{x}=1$

Более половины этого значения обеспечивала сумма пяти факторов, признанных наиболее влияющими на показатель энергоэффективности предприятия:

- удельная материалоемкость продукции - $X_{1}$, руб.;

- инвестиции в программы энергосбережения - $X_{2}$, млн руб.;

- выработка энергетических ресурсов собственными источниками энергии - $X_{3}$, доля от общего потребления;

- затраты на содержание энергоснабжающих сетей предприятия - $X_{4}$, млн руб.;

- объем выпуска продукции - $X_{5}$, млн руб.

Значения именно этих факторов будем использовать в качестве входных величин при синтезе нейронной сети.

Статистические данные десяти предприятий по пяти вышеуказанным факторам $X_{1}-X_{5}$ и энергоемкости Y представлены в Таблице 1.

Таблица 1. Влияющие факторы и энергоемкость предприятий

\begin{tabular}{|c|c|c|c|c|c|c|}
\hline № предприятия & $Y$ & $x_{1}$ & $x_{2}$ & $x_{3}$ & $x_{4}$ & $X_{5}$ \\
\hline 1 & 0.241 & 0.80 & 0.9 & 0.10 & 0.9 & 265.8 \\
\hline 2 & 0.163 & 0.68 & 0.8 & 0.30 & 1.9 & 670.0 \\
\hline 3 & 0.212 & 0.73 & 0.1 & 0.06 & 0.7 & 164.2 \\
\hline 4 & 0.261 & 0.79 & 0 & 0.03 & 1.1 & 124.1 \\
\hline 5 & 0.226 & 0.81 & 0.7 & 0.07 & 0.5 & 47.2 \\
\hline 6 & 0.184 & 0.76 & 1.3 & 0.15 & 0.3 & 37.2 \\
\hline 7 & 0.193 & 0.77 & 1.8 & 0.17 & 0.6 & 87.4 \\
\hline 8 & 0.242 & 0.78 & 1.0 & 0.13 & 0.5 & 58.7 \\
\hline 9 & 0.198 & 0.69 & 1.6 & 0.19 & 1.2 & 117.2 \\
\hline 10 & 0.173 & 0.66 & 2.6 & 0.70 & 3.9 & 9200.0 \\
\hline
\end{tabular}

При выборе вида ИНС учитываем, что для задач моделирования вещественных переменных оказывается эффективным применение нейросети типа прямонаправленный многослойный перцептрон [15]. Подходы к решению задач экономики с сетью данного вида были описаны автором в работе [8].

Структура данной сети предполагает наличие входного слоя, одного или нескольких внутренних (скрытых) слоев и выходного слоя. При выборе числа слоев исходим из целесообразности начать процесс синтеза сети с одного скрытого слоя, увеличивая их при необходимости.

Предлагаемая для синтеза нейронная сеть обозначается NN:5-n-1, что означает: нейронная сеть с пятью входами, $n$ нейронами скрытого слоя (количество которых может варьироваться) и одним нейроном выходного слоя (Рисунок 1). 


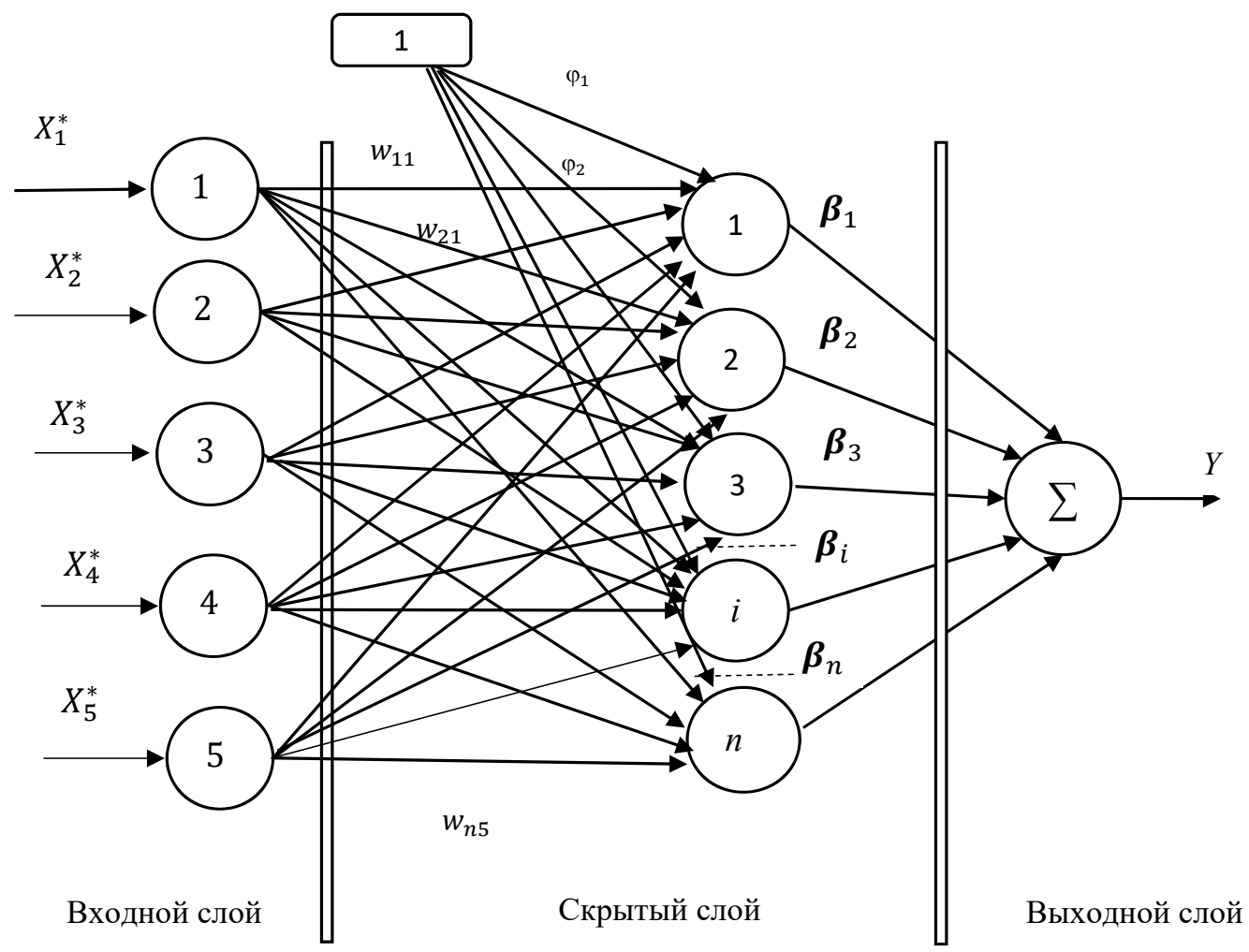

Рисунок 1. Нейронная сеть для управления энергоэффективностью предприятия

Функционирование ИНС происходит следующим образом. На первый слой подаются нормированные значения влияющих факторов

$X_{k}^{*}=\frac{X_{k}}{X_{6}}, \quad k=\overline{1,5}$,

где $X_{k}$ - величина входного фактора, $X_{6}$ - базовое значение фактора (как правило, наибольшее значение фактора, чтобы значение нормированной входной переменной $X_{i}^{*}$ находилось в диапазоне от 0 до 1).

Каждое из нормированных значений входного фактора умножается на весовой коэффициент wik, где $i$ - порядковый номер нейрона скрытого слоя (от 1 до n), a $\mathrm{k}$ - порядковый номер нейрона входного слоя. Поступившие на каждый нейрон скрытого слоя сигналы суммируются и сумма сигналов на каждом из них равна:

$$
n e t_{i}=\sum_{k=1}^{5} X_{k}^{*} w_{i k}
$$

т.e. $\quad n e t_{1}=X_{1}^{*} w_{11}+X_{2}^{*} w_{12}+X_{3}^{*} w_{13}+X_{4}^{*} w_{14}+X_{5}^{*} w_{15}$

$$
n e t_{2}=X_{1}^{*} w_{21}+X_{2}^{*} w_{22}+X_{3}^{*} w_{23}+X_{4}^{*} w_{24}+X_{5}^{*} w_{25} \text {, }
$$

$$
n e t_{i}=X_{1}^{*} w_{i 1}+X_{2}^{*} w_{i 2}+X_{3}^{*} w_{i 3}+X_{4}^{*} w_{i 4}+X_{5}^{*} w_{i 5},
$$

$$
n e t_{n}=X_{1}^{*} w_{n 1}+X_{2}^{*} w_{n 2}+X_{3}^{*} w_{n 3}+X_{4}^{*} w_{n 4}+X_{5}^{*} w_{n 5} .
$$


Суммарный входной сигнал каждого нейрона скрытого слоя также сдвигается на величину $\varphi_{i}$, т.е.

$x_{i}=$ net $_{i}-\varphi_{i}$.

(5)

Далее сдвинутый сигнал $x_{i}$ преобразуется активационной сигмоидальной функцией $\mathrm{f}(x)$, обеспечивающей нелинейное преобразование поступившего на нейрон сигнала (Рисунок 2). Таким образом, выход $i$-го нейрона срытого слоя сети $a_{i}$ равен

$a_{i}=f\left(x_{i}\right)$.

(6)

В матричной форме преобразование сигналов по соотношениям формул (4) - (6) представляется системой уравнений:

net $=\mathbf{w} \times \mathbf{X}^{*}$,

$\mathbf{a}=f($ net- $\varphi)$,

где net - вектор суммы сигналов, поступивших на нейроны скрытого слоя

$$
\text { net }=\left|\begin{array}{c} 
\\
n e t_{1} \\
\cdots \\
n e t_{i} \\
\cdots \\
\text { net }_{n}
\end{array}\right|
$$

$\mathbf{w}$ - матрица весовых коэффициентов при передаче сигналов от входного слоя к внутреннему

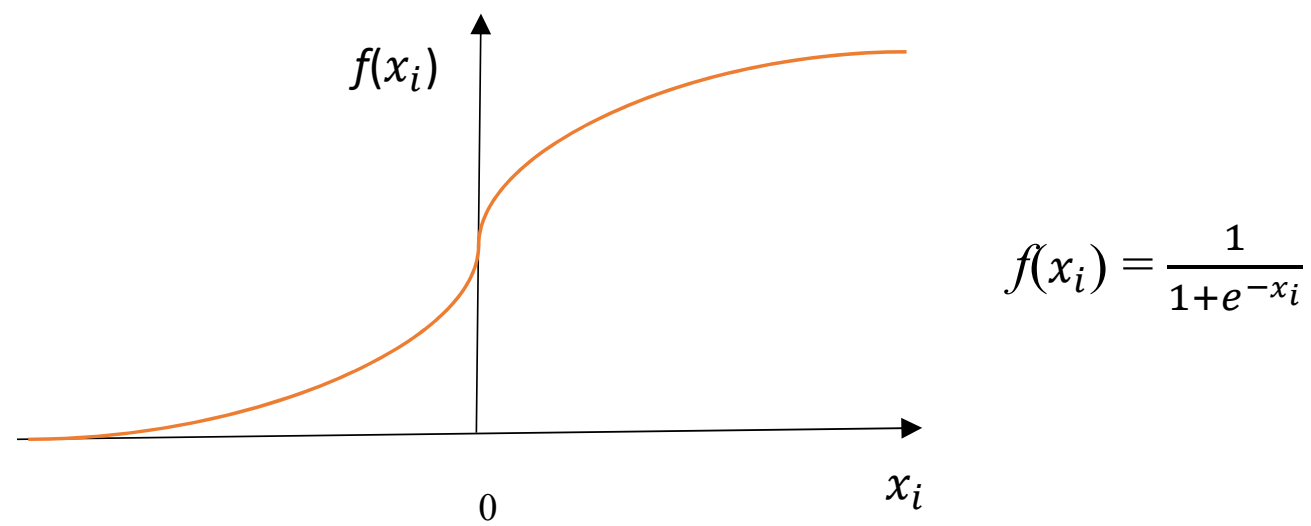

Рисунок 2. Сигмоидальная активационная функция 


$\mathbf{w}=\left|\begin{array}{ccccc}w_{11} & w_{12} & w_{13} & w_{14} & w_{15} \\ w_{21} & w_{22} & w_{23} & w_{24} & w_{25} \\ \ldots & \ldots & \ldots & \ldots & \ldots \\ w_{i 1} & w_{i 2} & w_{i 3} & w_{i 4} & w_{i 5} \\ \ldots & \ldots & \ldots & \ldots & \ldots \\ w_{n 1} & w_{n 2} & w_{n 3} & w_{n 4} & w_{n 5}\end{array}\right|$

$\boldsymbol{X}^{*}$ - вектор нормированных входных величин влияющих факторов

$$
\boldsymbol{X}^{*}=\left|\begin{array}{c}
X_{1}^{*} \\
X_{2}^{*} \\
X_{3}^{*} \\
X_{4}^{*} \\
X_{5}^{*}
\end{array}\right|
$$

a - вектор выходных сигналов нейронов скрытого слоя

$$
\mathbf{a}=\left|\begin{array}{c}
a_{1} \\
\cdots \\
a_{i} \\
\cdots \\
a_{n}
\end{array}\right|
$$

$\varphi-$ вектор сдвигов

$$
\boldsymbol{\varphi}=\left|\begin{array}{l}
\varphi_{1} \\
\varphi_{2} \\
\varphi_{3} \\
\varphi_{4} \\
\varphi_{5}
\end{array}\right|
$$

Выходное значение энергоемкости $Y$ определяется как сумма результатов расчетов нейронов скрытого слоя, умноженных на соответствующий весовой коэффициент, т.е.

$Y=\sum_{i=1}^{n} a_{i} \beta_{i}$

где $\beta_{i}$ - весовые коэффициенты передачи сигналов от нейронов скрытого слоя к выходному нейрону. 
Обучение (синтез) нейронной сети заключается в нахождении таких значений весовых коэффициентов $w i k, \beta i$ и коэффициентов сдвига $\varphi i$, которые бы обеспечивали удовлетворительное совпадение расчета сети на основе входных факторов и реальных значений энергоемкости.

Отметим, что, например, при 20 нейронах скрытого слоя оптимизации подлежит 140 значений величин $w i k, \beta i$ и $\varphi \mathrm{i}$, а при 50 нейронах - 350. Решение этой непростой задачи стало возможным благодаря компьютеризации процесса обучения.

Обучение, т.е. подбор значений вышеуказанных параметров осуществляется многократным повторением расчетов с изменением коэффициентов по определенному алгоритму. Весьма часто в качестве алгоритма расчета параметров нейросети персептронного типа применяется метод обратного распространения ошибки, однако известен недостаток этого метода - «зависание» в точках локальных экстремумов.

В настоящей работе синтез нейронной сети осуществлялся при помощи генетического алгоритма. Сущность данного метода применительно к решаемой в настоящей статье задаче описана в [10]. Данный метод копирует законы селекции, наблюдаемые в природе. «Хромосомами» при этом являются наборы значений весовых коэффициентов и коэффициентов сдвига, представляемые в двоичном коде и расположенные в строго определенной последовательности. Каждый весовой коэффициент является «геном» хромосомы. Набор «хромосом», подлежащих «попарному скрещиванию», представляет «популяцию».

Совокупность «хромосом» в популяции со стохастически определенными значениями «генов» являются «родителями», каждый их которых имеет свой номер. Как и в природе, стохастически определяются номера скрещиваемых родителей и номера ячеек, по которым происходит «скрещивание». При «скрещивании» образуется новая хромосома, состоящая из частей хромосом родителей. Способ образования новой хромосомы-«потомка» различен: кроссовер, инверсия, мутация, реализуемые с вероятностью, близкой к наблюдаемой в природе [10].

Для каждой из «хромосом» рассчитывается выходное значение нейронной сети (в нашем случае значение энергоемкости) и определяется по выбранному критерию близость совпадения результата со статистическим значением. Из полученной после серии скрещиваний пар «хромосом» (после эпохи обучения) исключается некоторое число хромосом, обеспечивающих наихудшее значение критерия приближения расчетного значения $Y$ к статистическому, так чтобы сохранилось установленное для популяции количество хромосом.

Стохастический процесс скрещивания многократно повторяется до тех пор, пока для анализируемых предприятий не будет подобрано такое сочетание значений весовых коэффициентов и коэффициентов сдвига, которое при расчете на статистических данных входных факторов будет обеспечивать приемлемое совпадение соответствующих им статистических значений энергоемкости.

В качестве обучающей выборки были использованы данные первых семи предприятий из исследуемой базы данных, а оставшиеся три предприятия (с 8 по 10) используем как тестовую выборку для проверки адекватности функционирования нейронной сети.

Для синтеза нейронной сети в соответствии с поставленной в настоящей статье целью была написана программа на языке FreePascal с использованием IDE Lasarus, осуществляющая следующие функции: нормирование статистических данных входных факторов, формирование структуры нейронной сети (выбор количества нейронов в скрытом слое), вид активационной функции, оптимизация параметров ИНС по описанному выше алгоритму в соответствии с выходными статистическими данными энергоемкости. Критерием останова оптимизационного процесса установлено заданное абсолютное значение разности между статистическим значением энергоемкости и рассчитанным. Автор выражает большую признательность старшему преподавателю НТУ «ХПИ» И. Обручу за помощь при составлении программы. 
Применение вышеописанной методики к решаемой в данной статье задаче осуществлялось при следующих параметрах:

- $\quad$ число нейронов скрытого слоя - 50;

- число хромосом в популяции - 100;

- коэффициент давления отбора (количество хромосом, исключаемых из обучения за одну эпоху) - 10\%;

- $\quad$ родительская группа - 100\%;

- $\quad$ характеристики скрещивания: кроссовер - 1, вероятность мутаций - 0.0004, вероятность инверсии 0.0002 , вероятность транслокации -0.0005 .

С целью определения быстроты сходимости процесса синтеза нейросети для установления рационального объема расчетов было принято заведомо большое число эпох, равное 1 млн, а также количество нейронов скрытого слоя, равное 50. Синтез нейронной сети потребовал 1 час 40 минут (процесcop - Intel Core i72.600 CPU @ 3,40 ГГц, Socket 1155 LGA; память (O3У) - DDR3 16 Гб 1.333 МГц; видеокарта - Radeon RX 5808 Гб).

Поверочные расчеты показали, что для практических целей количество нейронов в скрытом слое может быть сокращено до 15-20, а число эпох ограничено 50.000. Это значительно сократит время синтеза ИНС, не повлияв существенно на ее точность. Быстроту приближения нейронной сети к сети, удовлетворяющей заданным значениям ошибки расчета, иллюстрирует Рисунок 3.

Из Рисунка 3 видно, что на начальном этапе синтеза нейросети (до 50.000 эпох) приближение к заданному критерию происходит весьма быстро, а при увеличении количества эпох от 450 тыс до 1 млн снижение погрешности происходит незначительно.

Для удобства синтеза нейронной сети программа Mendel4 содержит окно ввода и вывода данных (Рисунок 4).

Результаты расчета энергоемкости предприятий на основе данных входных факторов с использованием синтезированной нейронной сети персептронного типа представлены в Таблице 2.

Таблица 2. Значения входных и выходных данных

\begin{tabular}{|c|c|c|c|c|c|c|c|c|}
\hline \multirow{2}{*}{$\begin{array}{c}\text { № } \\
\text { предприятия }\end{array}$} & \multicolumn{5}{|c|}{ Входной вектор } & \multirow{2}{*}{$\begin{array}{c}\text { Статистическое } \\
\text { значение } Y\end{array}$} & \multirow{2}{*}{$\begin{array}{c}\text { Выход } \\
\text { нейронной } \\
\text { сети }\end{array}$} & \multirow{2}{*}{$\underset{\%}{\text { Ошибка, }}$} \\
\hline & $X_{1}$ & $X_{2}$ & $x_{3}$ & $X_{4}$ & $X_{5}$ & & & \\
\hline 1 & 0.80 & 0.9 & 0.10 & 0.9 & 265.8 & 0.241 & 0.240 & 0.46 \\
\hline 2 & 0.68 & 0.8 & 0.30 & 1.9 & 670.0 & 0.163 & 0.163 & 0.26 \\
\hline 3 & 0.73 & 0.1 & 0.06 & 0.7 & 164.2 & 0.212 & 0.216 & 1.68 \\
\hline 4 & 0.79 & 0 & 0.03 & 1.1 & 124.1 & 0.261 & 0.261 & 0.08 \\
\hline 5 & 0.81 & 0.7 & 0.07 & 0.5 & 47.2 & 0.226 & 0.231 & 2.22 \\
\hline 6 & 0.76 & 1.3 & 0.15 & 0.3 & 37.2 & 0.184 & 0.181 & 1.44 \\
\hline 7 & 0.77 & 1.8 & 0.17 & 0.6 & 87.4 & 0.193 & 0.194 & 0.44 \\
\hline 8 & 0.78 & 1.0 & 0.13 & 0.5 & 58.7 & 0.242 & 0.198 & 18.38 \\
\hline 9 & 0.69 & 1.6 & 0.19 & 1.2 & 117.2 & 0.198 & 0.182 & 8.11 \\
\hline 10 & 0.66 & 2.6 & 0.70 & 3.9 & 9200.0 & 0.173 & 0.204 & 17.75 \\
\hline
\end{tabular}

Анализ результатов, представленных в Таблице 2, показывает, что построенная нейронная сеть с весьма высокой точностью рассчитывает результаты энергоемкости предприятий по статистическим данным, на которых проводился ее синтез. Так, из семи предприятий, попавших в обучающую выборку, по четырем погрешность расчета составила менее $0.5 \%$, у остальных трех - менее $2.5 \%$.

Как отмечалось в работе [4], нейронная сеть персептронного типа обладает обобщающими свойствами, позволяющими выявлять внутренние количественно неопределенные, характерные для объекта моделирования взаимосвязи, влияющие на выходную величину. Проанализируем по данным Таблицы 2 , в какой мере проявляется это свойство на примере статистических данных предприятий, не попавших в выборку при синтезе нейронной сети. 


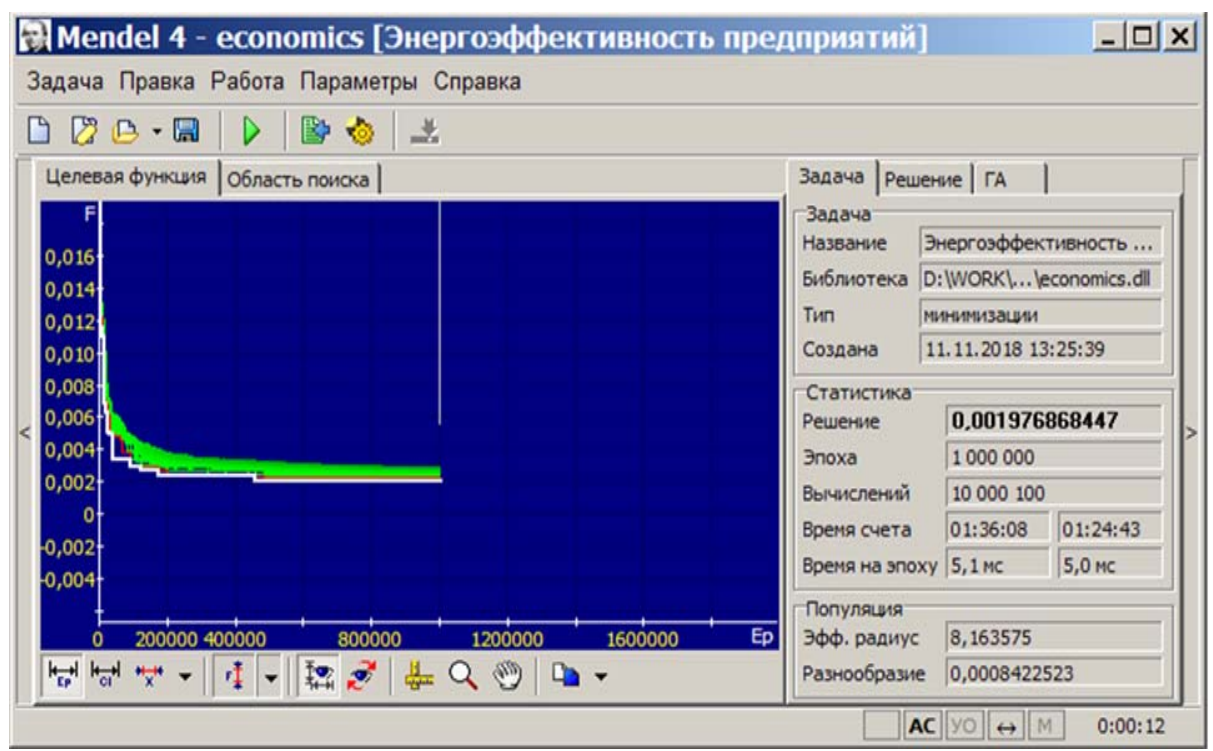

Рисунок 3. Процесс оптимизации весовых коэффициентов ИНС и сдвигов нейронов в программе Mendel4

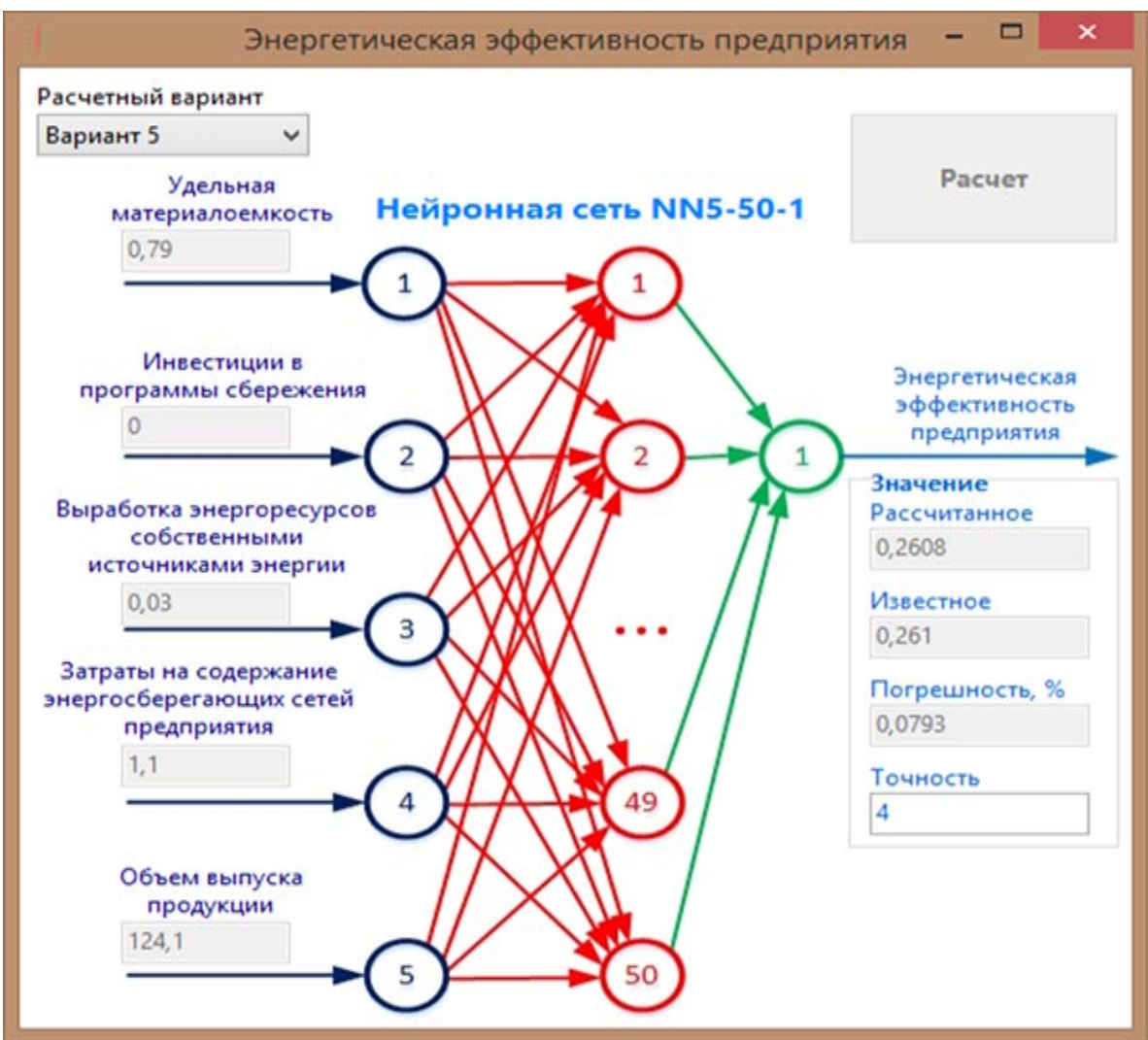

Рисунок 4. Окно ввода-вывода данных в программе Mendel4 
Погрешность расчетов показателя энергоемкости по предприятиям из тестовой выборки (8-10 в Таблице 2) находится в пределах 19\%, что существенно больше, чем на данных обучающей выборки. Учитывая дискретный характер стоимости оборудования, закупаемого для модернизации, а также других показателей, закладываемых в планы мероприятий при управлении энергоэффективностью, применение нейронных сетей для расчета показателя энергоемкости следует считать оправданным. Данные расчеты дают ориентировочное значение показателя энергоэффективности при определении целесообразной суммы средств на модернизацию.

Наличие значительных погрешностей может также указывать на наличие не выявленной специфики взаимосвязей, характерных для этих предприятий, и на целесообразность при использовании нейронных сетей для управления энергоэффективностью предприятий проведения кластеризации предприятий по типу и объему выпускаемой продукции, по характеру технологического процесса и т.п. В рамках одного предприятия имеет смысл пополнение числа выборок в разрезе ежегодных статистических данных с увеличением числа входных факторов, влияющих на показатель энергоэффективности данного предприятия.

Отметим, что разработка предложенной в настоящей статье методики применения нейронной сети основывалась на статистических данных, которые были использованы при создании методики на основе априорного ранжирования и корреляционно-регрессионного анализа [16]. Поскольку оба эти подхода направлены на достижении одной и той же цели - улучшение энергоэффективности предприятия, выполним их сопоставление.

Метод априорного ранжирования организационно более трудоемок, так как требует подбора достаточного числа квалифицированных экспертов. Кроме того, как отмечалось в [16], к его недостаткам относится определенная субъективность, поскольку при оценке тех или иных факторов эксперты пользуются своим прежним опытом или взглядами. Кроме того, в случае несоответствия критериям конкордации Кэнделла и Спирмена требуется анализ причин этого несоответствия, возможная замена состава экспертов и повторения расчетов.

При использовании предложенной методики на основе нейронных сетей все расчетные операции выполняются компьютером по разработанным алгоритмам.

Вышеуказанные подходы не противоречат, а дополняют друг друга. Методика, основанная на статистических методах, может быть использована для определения факторов, влияющих на повышение энергоэффективности предприятия. Методика, основанная на применении нейронной сети, позволяет получить приближенное значение показателя энергоэффективности при тех или иных значениях влияющих факторов.

\section{ВЫВОДЫ}

Применение при организации процесса управления энергоэффективностью предприятия нейронных сетей, синтезированных методом генетического алгоритма, целесообразно для получения приближенного значения прогнозируемой энергоемкости, что может быть использовано при определении объема средств, выделяемых на мероприятия, обеспечивающие снижение потребления энергии.

Проведенные на основе построенной нейронной сети персептронного типа расчеты продемонстрировали высокую точность прогнозирования значений энергоемкости для предприятий, которые были включены в обучающую выборку, и приемлемую погрешность при проверке по предприятиям, включенным в тестовую выборку. 
Показана целесообразность дальнейшего развития предложенной в статье методики моделирования энергоэффективности путем кластеризации промышленных предприятий по видам выпускаемой продукции, объему производства и другим факторам.

\section{REFERENCES}

1. Cabinet of Ministers of Ukraine (2017). Енергетична стратегія України на період до 2035 року «Безпека, енергоефективність, конкурентоспроможність» [Energetychna strategiya Ukrainy na period do 2035 roku «Bezpeka, energoefektyvnist', konkurentospromozhnist'»]. Retrieved from http://zakon5.rada.gov.ua/laws/show/605-2017-\%D1\%80

2. Denysiuk, S. P., \& Tarhonskyi, V. А. (2017). Сталий розвиток енергетики України у світових вимірах [Stalyi rozvytok enerhetyky Ukrainy u vsitovykh vymirakh]. Power engineering: economics, technology, ecology, 3, 7-28. https://doi.org/10.20535/18135420.4.2017.130871

3. Dzhedzhula, V. V. (2014). Енергозбереження промислових підприємств: методологія формування, механізм управління [Еnеrhozberezhennia promyslovykh pidpryiemstv: metodolohiya formuvannia, mekhanizm upravlinnia] (346 p.). Vinnytsia: VNTU.

4. Ezhov, A. A., \& Shumskiy, S. A. (1998). Нейрокомпьютинг и его применения в экономике и бизнесе [Neirokompiutingh i eho prymeneniya v ekonomike i biznese] (222 p.). Moscow: MIFI. Retrieved from https://docs.google.com/file/ d/0B7zVAgSBB3MeaWVZemVfcW1IN28/edit

5. Hornik, K., Stinchcombe, M., \& White, H. (1989). Multilayer feedforward networks are universal aproximators. Neural networks, 2, $359-366$.

6. International Energy Agency (2014). Energy efficiency indicators: fundamentals on statistics (387 p.). Retrieved from https://webstore.iea. org/energy-efficiency-indicators-fundamentals-on-statistics

7. Khan, T. F. (2016). Організаційно-економічне забезпечення ефективного використання енергетичних ресурсів на прикладі машинобудівних підприємств [Orhanizatsiyno-ekonomichne zabezpechennia efektyvnoho vykorystannia enerhetychnykh resursiv na prykladi mashynobudivnykh pidpryiemstv]. Extended abstract of candidate's thesis. Kharkiv: National Technical University "Kharkiv Polytechnic Institute".

8. Klepykova, S. V., \& Klepykov, V. B. (2005). К использованию метода нейронных сетей для решения экономических задач [K ispolzovaniyu metoda neironnykh setei dlia reshenyia ekonomicheskikh zadach]. Herald of the National technical university "KhPI", 31, 59-66.

9. Krichevskiy, M. L. (2016). Методы исследований в менеджменте [Metody issledovaniya v menedzhmente] (296 p.). Moscow: KnoRus.

10. Makhotylo, K. V. (2011). Диплоидный генетический алгоритм со смертностью [Diploydnyi geneticheskiy algoritm so smertnostiu]. Problems of management and informatics, 3, 138-150. Retrieved from http://repository.kpi.kharkov.ua/handle/KhPI-Press/30802

11. Makhotylo, K. V. Mendel - программа решения задач глобальной оптимизации [Mendel - programma resheniya zadach globalnoy optimizatsii]. Retrieved from https://sites.google.com/site/kvmahotilo/mendel

12. Matviychuk, A. V. (2011). Штучний інтелект в економіці: нейронні мережі, нечітка логіка [Shtuchnyi intelekt v ekonomitsi: neironni merezhi, nechitka logika] (439 p.). Kyiv: KNEU.

13. Mykytenko, V. V. (2004). Енергоефективність промислового виробництва [Enerhoefektyvnist promyslovoho vyrobnytstva] (281 p.). Kyiv: Obyednanyi instytut ekonomiky.

14. Ptuskyn, A. S. (2008). Нечеткие модели и методы в менеджменте [Nechetkiye modeli i metody v menedzhmente] (216 p.). Moscow: Izdatelstvo MGTU im. N. E. Baumana.

15. Rosenblatt, F. (1962). Principles of neurodynamics: perceptrons and the theory of brain mechanisms (616 p.). Washington: Spartan Books.

16. Sergeev, N. N. (2013). Оценка факторов, влияющих на энергетическую эффективность промышленных предприятий [Otsenka faktorov, vliyayushchykh na enerhetycheskuyu effektivnost' promyshlennykh predpriyatiy]. Economics and law, 2, 94-97. Retrieved from https://cyberleninka.ru/article/v/otsenka-faktorov-vliyayuschih-na-energeticheskuyu-effektivnost-promyshlennyh-predpriyatiy

17. Shidlovskiy, A. K., \& Kovalko, M. P. (Eds.) (2001). Паливно-енергетичний комплекс України на порозі третього тисячоліття [Palivno-energetichniy kompleks Ukrainy na porozi tretiogo tisyacholittya] (400p.). Kiyv: Ukrainski entsiklopedichni znannya.

18. Sukhodolia, O.М. (2010). Методологічні засади прийняття управлінських рішень у сфері енергоефективності [Metodolohichni zasady pryiniattia upravlinskykh rishen u sferi enerhoefektyvnosti]. Naukovyi visnyk Akademii munitsypalnoho upravlinnia. Seriya: Upravlinnia, 3, 17-24.

19. Technical Committee ISO/TC 301 (2018). Energy management systems - Requirements with guidance for use. Retrieved from https://www. iso.org/obp/ui/\#iso:std:iso:50001:ed-2:v1:en 